\title{
Future directions for protocol testing, learning the lessons from the past
}

\author{
Dr D. Rayner \\ National Physical Laboratory \\ Teddington, Middlesex, UK, TW11 OLW, phone: +44 181943 \\ 7040, fax: +44 181977 7091, e-mail: Dave.Rayner@npl.co.uk
}

\begin{abstract}
A reflection on the history of all aspects of protocol testing reveals both successes and failures, although many observers take a purely negative view. Important lessons need to be learned if protocol testing in the future is to be regarded in a more positive light. There are now new signs of hope in some the most recent developments. Drawing upon the lessons from the past and the current encouraging developments, this paper sets out to recommend the directions that should be taken in the future if the subject is to recover from its slump in popularity and its good prospects are to be realised.
\end{abstract}

\section{Keywords}

Protocol testing, conformance, interoperability, cost-effective testing 
After over 17 years in the protocol testing business, there is a lot to reflect back upon. By learning the lessons from the history of our subject we may more confidently predict the way which ought to be followed in the future. This paper sets out to present some of the more important lessons from the past, to take stock of where we are, and to point the way forward, more out of pragmatism than idealism.

\section{STANDARDISED CONFORMANCE TESTING}

\section{Lessons from the past}

The concept of standardised protocol conformance testing emerged out of the strong collective desire for Open Systems Interconnection (OSI) in the early 1980s. It was widely assumed that there would be an enormous market for OSI products from many different vendors and that the only way confidence could be given in the interoperability of these products would be to give an assurance that they met all the requirements of the relevant protocol standards. This meant that conformance testing was considered necessary for the success of OSI and it was assumed that such testing would be required by public procurement agencies.

Given this motivation, the OSI conformance testing methodology and framework standard was developed as a five-part standard published in 1991 and 1992, with second editions and two extra parts being published three years later (ISO/IEC 9646, 1994 and 1995). This standard was a tremendous achievement, the result of a consensus painstakingly developed among representatives of testing laboratories, major suppliers, network operators, researchers, and consultants representing users. At the beginning in 1983 there was considerable mutual mistrust between the suppliers and testing laboratories, but gradually each came to understand the other's point of view and they began to work together constructively towards the common goal: an agreed methodology and framework for objective, standardised, protocol conformance testing. The result was the most comprehensive testing methodology standard ever produced in any area of information technology, published not only by ISO/IEC but also by ITU-T and CEN.

Unfortunately, this methodology was developed as an afterthought in the whole OSI enterprise, and its developers were seen as a separate group of testing specialists unconnected with the protocol specifiers. Hence, the testing specialists were forced to try to apply their testing methodology to protocols that had been developed with no thought for testability. Guidance given on how to produce more testable protocols was largely ignored. Furthermore, those who ended up developing the standardised test suites and related standards were separate from 
those who had developed each of the protocols. Thus, there was a lack of real understanding of the protocols which meant that the test designers were unable to make informed decisions to improve the cost-effectiveness of the test suites; instead they tended to apply the methodology in a rather mechanical unimaginative, even unintelligent way. This was compounded by the fact that there was no feedback loop - no possibility of the work of the test designers feeding back into improvements to the protocol designs. With hindsight, we can see that it was almost inevitable that the test suites would be too large, too expensive and insufficiently focused on real market needs - in short, not at all cost-effective.

Standardised conformance testing was thought to be applicable uniformly to all protocols which fitted within the OSI seven-layer architecture, apart from those protocols in the Physical layer which concern the signals relevant to a particular physical medium (e.g. electrical, optical or radio signals). Little thought was given to the question of when to standardise and when not to, nor to the question of when the coverage of the test suite needed to be comprehensive and when it could be rather more superficial or patchy. Furthermore, if it was suggested that the methodology should be applied to a non-OSI protocol, the tendency was to adapt the protocol to OSI rather than to adapt the methodology to non-OSI protocols.

\section{Current situation}

Although a lot of conformance test suites have been produced, relatively few in the voluntary sphere (outside telecoms) have been standardised internationally, and even fewer are being used or properly maintained. In contrast, in the telecoms field, especially in the regulatory sphere, standardised test suites are being produced, used and maintained, particularly by ETSI.

There is a move now towards more flexible application of the methodology. It is being applied with appropriately focused coverage to suppliers' needs for development testing. It is being adapted to apply to non-OSI protocols. It is being enhanced to meet the more stringent requirements of testing security protocols, using what is called "strict conformance testing" (see Barker, 1997).

\section{The way forward}

There is much to value and hold on to in the conformance testing methodology and framework, but it must be applied flexibly and with appropriate adaptation to meet the market needs in each case in the most cost-effective way. Sometimes there will still be a requirement for standardisation of test suites, e.g. to meet regulatory requirements or the needs of high-risk areas like security or safety-critical software, but often standardisation will be unnecessary. Coverage should be chosen to be appropriate to match the risks associated with a failure to conform to the requirements of the protocol standard. To achieve this protocol designers and/or product implementors must be involved in the test design process.

There should also be provision for feedback from the test design process into improved protocol design, but more of this in the next section. 


\section{PROTOCOL DESIGN AND TESTABILITY}

\section{Lessons from the past}

A major contributory factor in the failure of OSI was the fact that most of the protocols were too complex, with too many options, too hard to implement, and too hard to test. Profiles were invented to try to overcome the complexity by making appropriate consistent selections of options from a stack of protocols. Hence, profiles were intended to solve the problem caused by the failure to design the protocols properly in the first place. Thus, this was tackling the symptom rather than the cause of the problem. Unfortunately, it made matters considerably worse, because there were too many profiles, each with still too many options, and the consequential protocol profile testing methodology (ISO/IEC 9646-6, 1994) was far too complicated and expensive to operate.

Since the early 1980s it has been said by some that there should be a formal description for each protocol standard. This could then be used to validate the protocol and would provide the basis for automated test generation. The reality was rather different. Firstly, there were too many different formal description techniques, whose advocates seemed to spend more time attacking the others than promoting the use of formal techniques. Secondly, the vast majority of people involved in protocol development could not understand the formal description techniques and would not trust the few who did understand them. Thirdly, there was inadequate tool support for the techniques. Fourthly, such formal descriptions as were developed were produced as an afterthought by academics, without adequate contact with the original protocol specifiers, usually without the full functionality of the protocol, and with no intention of maintaining the specification in line with the standard.

\section{Current situation}

It is now accepted that we must learn from the success of Internet, in which protocols are kept simple with very few options, and are implemented before being finalised, to demonstrate ease of implementation and understandability of the specification.

Profiles are now largely seen as irrelevant. Where a stack of protocols is to be tested together, it now seems more appropriate to use interoperability testing rather than profile conformance testing with all its complexity and cost.

Some significant progress has been made in the acceptance of formal specifications. Firstly, the OSI Distributed Transaction Processing protocol was standardised in 1992 and revised in 1996 (ISO/IEC 10026-3, 1996) including two annexes giving informative formal descriptions in LOTOS and Estelle. Although they were only informative these specifications were authoritative and accepted by the protocol defining group as being complete descriptions of the protocol. There was even clause by clause parallelism between the text and the LOTOS specification. Unfortunately, this protocol failed to be widely accepted by the 
market, and soon afterwards both LOTOS and Estelle fell largely into disuse in the protocol standardisation community in ISO/IEC JTC1.

Secondly, SDL (ITU-T Z.100, 1994, and ITU-T Z.105, 1994) has now become the dominant formal description technique within the protocol standardisation community, increasingly used in ETSI and ITU-T. A breakthrough came when an ETSI committee helped ITU-T develop the text and SDL specifications in parallel for INAP (Intelligent Network Application Protocol) CS-2, as specified in ITU-T Q.1224 (1997) and ITU-T Q.1228 (1997). This resulted in the formal specification being published by ITU-T as a normative annex to the standard, although in cases of conflict the text takes precedence over the SDL. Tools were used to perform protocol validation at various stages during the development. The result was faster, cheaper and better quality protocol development.

\section{The way forward}

What is needed is to build upon the INAP CS-2 experience, leading to the parallel development of text and SDL becoming the norm, with the SDL becoming the normative specification, with much of the text just having the status of informative commentary on the SDL. There will, however, probably always be a need for some normative text to express those requirements that are hard to express in SDL and to avoid the SDL having to become too detailed and complex. The SDL specification should then become the basis for validation, animation and automated test generation. It could also, if appropriate, be the basis for production of a trial or reference implementation, but experience shows that such an implementation is likely to require some non-trivial hand-coding and therefore even an implementation derived from the SDL is likely to need testing.

Moreover, the protocol design process should aim to minimise complexity, building in testability, focusing requirements on what is really needed to achieve interoperability, and including trial implementation before finalisation of the specification.

In order to achieve these objectives, it is important that future protocol design groups should include all the expertise necessary to do the job properly. This includes expertise in protocol design, the intended field of use, testability, testing methodology, formal specification using SDL, other supporting techniques (e.g. state tables, ASN.1, message sequence charts), and use of relevant software tools. It should be stressed that protocol designers can now invest with confidence in the training necessary to become knowledgeable in all the key techniques (testing methodology, TTCN, SDL, ASN.1, MSCs) because of their maturity and stability. 


\section{TEST SUITE DESIGN}

\subsection{Test purposes and test suite coverage}

\section{Lessons from the past}

Perhaps the biggest problem with ISO/IEC 9646 has been its guidance on the development of test suite structures and test purposes. The importance of test purposes is not in doubt, for they provide a simple, easy to understand description of what a test case is to achieve. They provide the appropriate level at which to discuss test suite coverage. They also facilitate an understanding of the verdicts produced when the derived test cases are run. The problem is that if the guidance given in ISO/IEC 9646-2 (1994) is followed mechanically, without any consideration for which test purposes are likely to be most effective, then it is all too easy to produce a test suite which is much too large, with many test purposes that are frankly irrelevant to achieving interoperable products.

\section{Current situation}

Test purposes are now playing a vital role in automated test generation. It is now increasingly accepted that automated test generation should not simply go directly from a formal specification of the protocol to the output of a test suite. Instead automation can be used in two stages, either separately or in combination. The first stage is the production of the test purposes from the formal specification, performed with appropriate parameterisation and interaction with the test designer. The test designer can then review the test purposes and make adjustments as necessary, possibly altering the parameterisation and going through the process several times until an acceptable set of test purposes is produced. Test purposes output from this process could either be written in stylised natural language or in Message Sequence Charts (MSCs) as specified in ITU-T Z.120 (1993). They could be designed for standardisation or for development testing of a specific product.

The second stage is to use test purposes as the input to test case generation, ensuring that only the desired test cases are generated. For this process the test purposes need to be expressed in a suitable formalism; MSCs seem to be a natural and increasingly popular choice. The test purposes for this second stage may have been generated using an automated tool or may have been hand-written.

\section{The way forward}

Much more guidance needs to be produced on adopting flexible approaches to deciding on test suite coverage. There needs to be informed analysis of where the practical problems and threats to interoperability lie in implementing a particular protocol and what therefore most needs to be tested. It needs to be decided what the overall objective of the test suite is and the coverage needs to be appropriate to achieving that objective. 
In some cases, a very small number of test cases will suffice, each perhaps with a high-level test purpose requiring a long sequence of state/event pairs to be exercised. In such cases, they are probably best identified by product developers getting together to share their knowledge of where the problems lie in implementation, just as they do to produce interoperability test suites for EuroSInet.

In other cases, a rather larger number of test cases may be needed, but perhaps skewed to focus on the most important features of the protocol. Only in a minority of cases should it be expected that large test suites with very even coverage of rather narrowly focused, atomic test purposes would be appropriate.

The development of coverage metrics could be useful, provided that they are used to inform the test designer rather than dictate what coverage must be used.

Automated test purpose generation will be useful in those cases where large test suites are still required, or where in development testing it is necessary to continually change the tests to focus on different problems in the implementation being tested; but for the development of very small test suites that are not continually changing such tools are probably unnecessary. Automated test case generation from test purposes should soon progress to the point where the only serious limitation to its use will come from the availability of authoritative SDL specifications, but before long even the lack of an SDL specification should not prove to be a limitation because practical automated test generation techniques will be applied to the extended finite state descriptions that are common in almost all protocol specifications. For input to such tools, we can expect that MSCs should become the dominant formalism for expressing test purposes.

\subsection{Test cases}

\section{Lessons from the past}

An abstract test case is a detailed implementation of a test purpose using a particular test method and expressed in a test specification language. The test method will be chosen from the set of test methods defined in ISO/IEC 9646-2 (1994) which has stood the test of time.

Abstract test cases are invariably written in TTCN (the Tree and Tabular Combined Notation, ISO/IEC 9646-3, 1992). Although TTCN is clearly the product of committee compromise, containing various arbitrary restrictions and inconsistencies, it has proved itself to be a widely applicable test specification language. There has been considerable investment in the development of TTCN tools and, although there were serious problems in the past with the quality of the tools, these problems have now been overcome.

The finalisation of the second edition of TTCN has been a long drawn-out process, caused primarily by the fact that all the members of the ISO/IEC editing group lost their funding for the work. This shows up a serious weakness in the voluntary standardisation process: if all the key people lose their support for the 
work, then any standards project can be brought to a halt. The risk of this happening is increased by the long timescales on which international standardisation operates.

Automated test generation has been criticised as being impractical in the past, not only for leading to inappropriate test suite coverage, but also for producing rather incomplete test cases which require a lot of manual editing.

\section{Current situation}

The second edition of TTCN is now technically complete and has undergone a process of validation by several European TTCN tool suppliers and others in the TTCN community in ETSI. A review copy (ISO/IEC 9646-3, 1997) was sent in early May to ISO/IEC JTC1/SC21 for national body and liaison organisation review. In parallel, the editor, Os Monkewich, is performing the final editorial work, including production of contents list and indexes. The main new features of the second edition are the use of ASN.1 94 (ISO/IEC 8824-1, 1994), concurrency, encoding operations, formalised test suite operations, modularity, and active defaults. Concurrency is useful not only for multi-party test cases, but also to provide structuring for multi-protocol or embedded test cases by using a different test component for each protocol used in the test case.

There is now a healthy competitive market in TTCN tools, especially in Europe, with most tools now supporting the main features of the second edition. The tools include editors, error checkers, compilers, interpreters, test case generators, test case validators, and behaviour simulators.

For some time, EWOS and ETSI have produced guidance documents related to TTCN, especially the TTCN style guide (ETG 25, 1994). ETSI is now involved in producing guides on the use of the second edition of TTCN and on the use of TTCN with ASN.1 94. Amongst this guidance there is expected to be guidance on relaxing the definition of a point of control and observation (PCO) to allow for communication with the test system if necessary, which is allowed by TTCN but not by ISO/IEC 9646-2 (1994).

\section{The way forward}

There now needs to be a period of consolidation based upon the complete second edition of TTCN. All the TTCN tools need to be brought into line with it. The ETSI guidance documents related to the second edition need to be completed and given widespread distribution. An effective maintenance process needs to be established for TTCN, given that there is nobody left in ISO/IEC JTC1/SC21 to maintain the standard after it is published.

It is clear that to be successful, automated test generation must produce good quality complete TTCN test cases, including declarations, constraints, preambles and postambles, as well as the test bodies. 


\section{TEST SYSTEMS}

\section{Lessons from the past}

The requirements of ISO/IEC 9646-4 (1994) regarding test systems largely concern the information to be stored in the conformance log. These requirements have proved to be valuable in giving a common basis of objective information on which to base a test report. If statements in a test report are questioned, they can usually be substantiated by looking at the relevant part of the conformance log.

The main problem with test systems has been the difficulty and cost of mapping the TTCN abstract test cases into the appropriate executable test cases. The problem is especially bad in those test systems which use points of control and observation different from those used in the abstract test cases.

\section{Current situation}

There is now a trend towards support for TTCN in test systems, both in providing a TTCN compiler or interpreter and in providing results analysis directly related to the TTCN. The European Commission's INTOOL projects have recently delivered a set of interface specifications which when implemented will enable tools from different suppliers to be used in combination. Perhaps the most important of these is the GCI interface (generic TTCN compiler/interpreter interface) which enables a single TTCN compiler or interpreter to be used with multiple test systems.

Another recent advance is the publication by ETSI of the TSP1 (test synchronisation protocol 1) specification (ETR 303, 1997). TSP1 should be usable as a general mechanism for implementing test coordination procedures.

\section{The way forward}

There seems to be no need to modify the requirements on conformance logs, but online interpretation of the logs in terms of the TTCN should become the norm. Test system development should concentrate on implementing the GCI interface in order to allow a TTCN front-end to be provided to each test system. This will avoid the need to spend a lot of effort translating TTCN into executable test languages and in maintaining the alignment between abstract and executable test suites. TTCN compilers need to be further developed to support more fully the second edition of TTCN. Care should be taken to ensure that they make full use of PICS (protocol implementation conformance statement) and PIXIT (protocol implementation extra information for testing) information, to minimise the need for interaction with the test engineer during compilation.

Further work should be done to investigate the practicality of using ETSI's TSP1 (ETR 303, 1997), the interface specifications from the Open Testing Environment INTOOL project, and any other potentially useful APIs (application programming interfaces) to improve flexibility and cost-effectiveness in the use of test tools. 


\section{TESTING SERVICES}

\subsection{Conformance testing}

\section{Lessons from the past}

The whole conformance assessment process specified in ISO/IEC 9646-5 (1994) has proved to be very robust. It is widely accepted as providing the definitive basis on which objective conformance testing services should be run, not least by accreditation bodies in their assessment of testing laboratories offering protocol testing services.

The interpretation of accreditation requirements in information and communication technologies (ICT) has needed internationally accepted guidance. This is because the traditional concepts of calibration and traceability which work well in areas involving physical measurement are not applicable to software and protocol testing in ICT. In their place the concept of validation of test tools had to be developed. Furthermore, guidance was also necessary to help determine which types of testing were sufficiently objective to be accreditable. A key idea in this was that the results should be repeatable at the one testing laboratory and reproducible by other testing laboratories.

\section{Current situation}

The interpretation of accreditation requirements in the ICT field has been agreed and published as ISO/IEC TR 13233 (1995). Its forerunner in Europe, ELA-G5 (1993), ought to be updated to align it to ISO/IEC TR 13233, but this is not a high priority at present for either the accreditation bodies or the European Commission.

For several years accreditation of protocol testing services was on the increase, coupled in Europe with the growth of agreement groups under ECITC (the European Committee for IT Testing and Certification). Now, however, because of the lack of a market demand for third party protocol conformance testing services in the voluntary sphere, the number of accredited laboratories has declined and ECITC agreement groups are disappearing. What are left are mostly testing laboratories operating in the telecommunications area, primarily in the regulatory sphere, plus a few offering testing services aimed at the US government procurement market.

\section{The way forward}

The problems of how to accredit protocol testing services are now solved, but in future such accreditation will be applied primarily in the telecommunications field. In addition, we can forecast a growth in security testing services, including the testing of security protocols. Given that the risks of non-conformity are much higher in the security field than in most other areas of ICT, and given that increased used of testing within security evaluation can reduce the costs and timescales to meet more closely the needs of industry, we can foresee the need for 
accredited "strict conformance testing" services. There is likely also to be a growth in safety-critical software testing, for similar reasons, but the protocol testing component of this is much less well developed. Thus, further research and development is needed in this area.

\subsection{Interoperability testing}

\section{Lessons from the past}

There have been two main approaches developed and applied to the provision of interoperability testing, but there is no standardised methodology. One approach was developed by SPAG (the Standards Promotion and Application Group), called PSI (process for system interoperability). In this approach, interoperability testing was an additional step that followed thorough conformance testing. The interoperability testing was based upon an SIS (system interoperability statement), which played a similar role to the PICS for conformance testing. It was conducted in a thorough and objective manner. The whole scheme was regarded by many as effectively an expensive certification scheme which was a long way from meeting market needs. As a result it never really took off, even though it was taken up both by JITC (the Joint Interoperability Testing Center) in the USA and by X/Open.

In contrast, there is the approach used by EuroSInet and other regional members of OSI ${ }^{\text {one }}$. For years they paid lip service to the idea that suppliers who engaged in testing their products in the interoperability testing workshops should have first ensured that their products had passed conformance testing. In reality, however, suppliers simply brought pre-release products into a cooperative multi-vendor testing workshop in order to conduct tests which could never be performed in their own laboratories. The concept was simple and the cost was very low. Product developers got together to agree what scenarios they wanted to test. These were written down in much the same way as conformance test purposes. They would then get together for a week of cooperative testing, performing pairwise tests with a large matrix on the wall showing who had tested with who, and was currently testing with who. Originally physical interconnection might have been via wide area networks or local X.25, but more recently Ethernet local area networks (LANs) have been used instead. Two problems emerged: the suppliers were very reluctant to publish the results thereby reducing the visibility of the activity to users and procurers; and as the membership changed from large multi-national suppliers to small niche market players, EuroSinet got locked into only applying its testing approach to X.400 and X.500 testing. The problem of publication of results was overcome by EuroSInet publishing a sanitised version of the report of the workshop, showing who tested with who and what was tested, but withholding the detailed results as these only applied to pre-release versions of the products; any bugs found should be corrected before product release.

Other approaches were advocated, notably by the European Commission CTS-4 interoperability testing project. Also EWOS published guidance documents 
including a vocabulary, a classification scheme, and a survey of what was happening around the world. However, these all remained rather theoretical.

\section{Current situation}

With PSI effectively dead, the only practical interoperability testing scheme is that still being used by EuroSInet. EuroSInet requested EOTC (the European Organisation for Testing and Certification) support to apply the approach more widely, but in the absence of even a clear timetable for a response EuroSInet got together with the EWOS Expert Group on Conformance Testing (EGCT) to propose a way forward. EWOS set up a project team (number 34) on interoperability testing, including involvement from the chairman of EuroSInet and the chairman of OSI ${ }^{\text {nee }}$. This produced a comprehensive report that led to the development of two specific project proposals which were approved by the EWOS Technical Assembly in December 1996.

One was for the production of a new set of guidance documents on interoperability and cost-effective testing, including the development of new ideas on built-in interoperability and built-in testability. There would also be work on an interoperability testing framework, SIS proformas, interoperability test suites, and pilot trials of the new ideas in three areas relevant to the GII (global information infrastructure). This project did not, however, go ahead because of a lack of funding available to EWOS.

The other project proposal was for the establishment of interoperability and capability demonstration facilities. This was essentially the idea of applying the EuroSInet interoperability testing concept across the whole scope of EWOS activities, but extending it to use the same low cost multi-vendor set up to demonstrate the capabilities of products based on EWOS specifications to potential users and procurers. This project did at least go ahead to the extent of holding a first interoperability testing workshop in Brussels in May 1997, focused on X.500 testing, to demonstrate the practicality of the idea and to advocate its use across the scope of whatever organisation takes over from EWOS later this year.

\section{The way forward}

A cost-effective testing approach should be developed based on bringing together the best of both conformance testing and interoperability testing to provide a consistent approach to testing that can be applied right through the product lifecycle. It should be flexible enough to cater for the needs of development testing, independent conformance testing, multi-vendor interoperability testing, operational monitoring and diagnostic testing, and regression testing. The concepts of built-in interoperability and built-in testability should be developed to see whether they can make a practical contribution to cost-effective testing.

Built-in interoperability means that the protocol design enhances rather than diminishes the prospects for successful interoperability of different suppliers' products. This implies keeping the number of options to a minimum and ensuring 
that the requirements expressed are all necessary to achieve successful interoperability.

Built-in testability means including features within the protocol to provide a selftesting capability or to facilitate more control over the conduct of conformance or interoperability testing. The difficulty is to find an organisation to take the lead in such work, now that the future of EWOS activities is in doubt both with regard to the funding and the organisational stability.

Where simple multi-vendor low cost interoperability testing is appropriate, the EuroSInet approach should be adopted. According to need, any appropriate available physical network could be used in place of the Ethernet LAN.

\section{RECOMMENDATIONS}

To summarise, the following recommendations are made regarding the future direction of protocol testing:

a) Standardised conformance test suites are only necessary in regulatory areas and in areas of high risk, like security and safety-critical software.

b) Test suite coverage should be chosen to match the risks of not testing.

c) Protocol designers, test designers and product implementors all need to work together to improve the effectiveness of protocol specifications and test specifications.

d) Normative SDL specifications should be developed together with the text description of telecoms protocols, and the SDL should be the basis for validation, animation, reference implementation, and automated test generation.

e) The protocol design process should aim to minimise complexity, building in testability, focusing on the essential interoperability requirements, and getting feedback from trial implementation before finalisation.

f) Protocol design groups should include expertise on testing methodology, TTCN, SDL, ASN.1, and MSCs.

g) Test purposes should be expressed in MSCs.

h) Automated test purpose generation should be developed for use in those cases where large test suites or continually changing test suites are required.

i) Automated test case generation from test purposes should be developed to start from either SDL or an extended finite state description and to produce complete TTCN test cases.

j) All TTCN tools and test suite developments should be aligned to the second edition of TTCN.

k) Test systems should either directly support TTCN input or should support the GCI interface so that they can be used with TTCN compilers or interpreters.

1) The practicality and cost-effectiveness of using TSP1 and other APIs should be investigated. 
m) ISO/IEC 9646-5 and ISO/IEC TR 13233 provide a sound basis for the accreditation of protocol testing laboratories.

n) Accredited testing will mainly be needed to meet regulatory requirements or the requirements of high risk areas, like security and safety-critical software.

o) The use of strict conformance testing should be encouraged in the security area, to reduce costs and timescales and thereby be more attractive to industry.

p) A cost-effective testing approach should be developed, based on the best of conformance and interoperability testing.

q) Flexible cost-effective testing should be applied consistently right across the product life-cycle.

r) The concepts of built-in interoperability and built-in testability need to be studied to determine their practicality and cost-effectiveness.

s) The EuroSInet approach to interoperability testing should be applied wherever low cost focused multi-vendor interoperability testing is needed.

\section{REFERENCES}

Barker, R.M., Hehir, S., Kelly, G., Lampard, R.P., Lovering, A., Parkin, G.I., Stevens, M.J. and Szczygiel, B.M. (1997) Strict conformance testing, NPL.

ELA-G5 (1993) Interpretation of accreditation requirements in ISO/IEC Guide 25 and EN 45001 - Guidance for information technology and telecommunications testing laboratories for software and communications testing services, WELAC and ECITC, Brussels.

ETG 25 (1994) The TTCN style guide, rev. 1, EWOS, Brussels.

ETR 303 (1997) Methods for testing and specification; Test synchronization; Architectural reference; Test synchronization protocol 1 specification, ETSI, Sophia Aritipolis, France.

ISO/IEC 8824-1 (1995) Abstract syntax notation one (ASN.1) Part 1: Specification of basic notation. Also ITU-T X.680.

ISO/IEC 9646-1 (1994) OSI conformance testing methodology and framework Part 1: General concepts. Also ITU-T X.290.

ISO/IEC 9646-2 (1994) OSI conformance testing methodology and framework Part 2: Abstract test suite specification. Also ITU-T X.291.

ISO/IEC 9646-3 (1992) OSI conformance testing methodology and framework Part 3: The tree and tabular combined notation. Also ITU-T X.292.

ISO/IEC 9646-3 (1997) OSI conformance testing methodology and framework Part 3: The tree and tabular combined notation. Mock-up for SC21 review, version 9.6.

ISO/IEC 9646-4 (1994) OSI conformance testing methodology and framework Part 4: Test realization. Also ITU-T X.293.

ISO/IEC 9646-5 (1994) OSI conformance testing methodology and framework Part 5: Requirements and guidance for test laboratories and their clients for the conformance assessment process. Also ITU-T X.294. 
ISO/IEC 9646-6 (1994) OSI conformance testing methodology and framework Part 6: Profile test specification. Also ITU-T X.295.

ISO/IEC 9646-7 (1995) OSI conformance testing methodology and framework Part 7: Implementation conformance statements. Also ITU-T X.296.

ISO/IEC 10026-3 (1996) Distributed transaction processing Part 3: Protocol specification.

ISO/IEC TR 13233 (1995) Interpretation of accreditation requirements in ISO/IEC Guide 25 - Accreditation of information technology and telecommunications testing laboratories for software and protocol testing services.

ITU-T Q.1224 (1997) Distributed function plane for intelligent networks capability set 2 .

ITU-T Q.1228 (1997) Interface recommendation for intelligent networks capability set 2 .

ITU-T Z.100 (1994) Specification and description language.

ITU-T Z.105 (1994) SDL combined with ASN.1.

ITU-T Z.120 (1993) Message sequence charts.

\section{BIOGRAPHY}

Dave Rayner joined NPL in 1975, became head of the protocol standards group in 1980 , head of the open systems group in 1993, and head of information systems engineering in 1996. In 1983 he became ISO rapporteur for OSI conformance testing, leading the development of ISO/IEC 9646 until the present day. From 1993 to 1996 he was convenor of ISO/IEC JTC1/SC21/JWG9 and editor of ISO/IEC TR 13233. From 1989 to 1996 he was chairman of the ECITC/OTL group, and during this time he edited ELA-G5. In 1993 he became chairman of EWOS/EGCT, a post he still holds. He assesses protocol testing laboratories for accreditation for UKAS, SWEDAC and DANAK. 